\title{
Leech Lattice Extension of the Non-Linear Schrodinger Equation Theory of Einstein Spaces
}

\author{
George Chapline \\ Lawrence Livermore National Laboratory, Livermore, CA, USA \\ Email: chapline1@1lnl.gov
}

How to cite this paper: Chapline, G. (2017) Leech Lattice Extension of the Non-Linear Schrodinger Equation Theory of Einstein Spaces. Journal of Modern Physics, 8, 2189-2199.

https://doi.org/10.4236/jmp.2017.814134

Received: October 13, 2017

Accepted: December 17, 2017

Published: December 20, 2017

Copyright $\odot 2017$ by author and Scientific Research Publishing Inc. This work is licensed under the Creative Commons Attribution International License (CC BY 4.0).

http://creativecommons.org/licenses/by/4.0/

\section{(c) (i) Open Access}

\begin{abstract}
Although the scalar nonlinear Schrodinger equation has provided valuable insights into how quantum mechanics might modify the classical general relativistic description of space-time, a detailed understanding of space-times with matter has remained elusive. In this paper, we propose generalizing the nonlinear Schrodinger equation theory of Einstein spaces to include matter by transplanting the $3+1$ dimensional theory to the 24-dimensional Leech lattice plus 1 time dimension. The scalar wave function and Chern-Simons gauge potential which encode the classical Kahler potential become $11 \times 11$ complex matrices belonging to a 195,442 dimensional representation of the Mathieu group $M_{11}$. This theory describes gravity coupled to internal degrees of freedom which include a supersymmetric $\mathrm{E}_{6} \times \mathrm{E}_{6}$ Yang-Mills theory of matter.
\end{abstract}

\section{Keywords}

Quantum Gravity, Quantum Space-Time, Emergent Space-Time, Space-Time Quantum Entanglement

\section{Introduction}

Among the known exact solutions of the Einstein's general relativity equations self-dual Einstein spaces stand out as particularly interesting [1]. Our interest is that these spaces were stimulated by the discovery that these solutions can also be expressed as solutions of the nonlinear Schrodinger equation in $2+1$ dimensions with a Chern-Simons gauge potential [2]. This in turn led to a "superfluid" representation for the vacuum state of a space-time with no matter [3] [4]. Independently of this development it had been suggested [5] [6] that a superfluid description of space-time might provide general insights into how quantum 
mechanics affects classical general relativity. It might be noted in this connection that, in contrast with the predictions of general relativity, there are theoretical [7] [8] [9] hints that in a quantum theory of gravity the surface of compact objects is not an event horizon. One possibility is that the surface of a compact object represents a phase transition in a superfluid theory of space-time [10]. If it turns out that the general relativistic description of compact objects is indeed flawed, the fault may well lay the likelihood that a consistent treatment of space-times with matter will require a quantum theory of space-time. Unfortunately, a quantum theory of space-times with matter has remained elusive up to the present time.

In this paper we wish to draw attention to the possibility that a matrix non-linear Schrodinger equation living on a Lorentzian extension of the 24-dimensional Leech lattice may provide the needed framework for a quantum theory of gravity and elementary particles in $3+1$ dimensions. Our basic idea is to replace the complex scalar wave function and gauge potential used in ref's [2] [3] to encode the Kahler potential of a self-dual Einstein space by complex matrices representing automorphisms of the complex Leech lattice. The Leech lattice was originally constructed as a result of its connection with perfect error correcting codes [11] [12]. From the point of view of constructing a quantum theory of space-time containing matter perhaps the most intriguing property of the Leech lattice is that there is a correspondence between the shapes of the minimal Leech lattice vectors (referred to hereafter as the "Leech polytrope") and the types of massless fields that occur in the 10-dimensional unification of supergravity and super Yang-Mills theories. For example, the number of minimal vectors with shape $\left(4^{2}, 0^{22}\right)$ is equal to $4 \times$ the number of components of a 2 -form field $B_{\mu \nu}$ in 24 -dimensions, while the vectors with shape $\left(-3,1^{23}\right)$ represent $2 \times$ the number of components of a gravitino field in 24 dimensions. An interesting feature of the Leech lattice is that if the Leech lattice generators with shape (8, $0^{23}$ ) - which are not vertices of the Leech polytope-are added to the vertices of the Leech polytrope, then the numbers of bosonic and femionic degrees of freedom are exactly equal. Remarkably the full automorphism group of the Leech lattice, the Conway group 0• [13], includes supersymmetry-like transformations between Leech polytrope vectors representing bosonic and fermionic degrees of freedom. Thus our Leech lattice set-up parallels the unification of supergravity and super-Yang-Mills interactions in 10-dimensions [14], with the caveat that the gravitational degrees of freedom are only active in the $3+1$ dimensions of ordinary space-time.

In order to breathe life into the matrix degrees of freedom associated with the Leech lattice, the wave function and gauge potential will be allowed to vary with position within a distinguished 2-dimensional hexagonal section of the Leech lattice. When time is added to the nonlinear matrix equation the wave function will depend not only on position within the distinguished 2-dimensional section of the Leech lattice, but also time. However if a self-duality condition is imposed 
on the wavefunction and gauge potential, then the time independent matrix nonlinear Schrodinger equation can be solved analytically, yielding soliton-like solutions that carry matrix internal degrees of freedom. If the holomorphic and anti-holomorphic are paired to provide a superfluid wave function for space-time, then the low lying excitations of the ground state will resemble the gravitational, anti-symmetric tensor, and elementary particle degrees of freedom that occur in 10-dimensional supergravity and super-Yang-Mills theories.

It seems though that the energy density of this emergent space-time will in general not be zero. Remarkably the numbers of bosonic and fermionic 2-form and Yang-Mills gauge field degrees of freedom in our theory exactly match. This means that at the level of $3+1$ dimensions the contribution of zero point fluctuations of the gauge fields to the ground state energy will vanish. However, the contributions of zero point fluctuations of the bosonic and fermionic gravitational degrees of freedom to the ground state energy will not exactly cancel each other. In addition, the direct interaction of self-dual and anti-self-dual solitons in the vacuum state will produce a nonzero energy density.

As discussed in more detail in Section 3, the symmetry group for the internal degrees of freedom in our model for space-times with matter will be $2: \mathrm{M}_{11}$, where $M_{11}$ is a sporadic permutation group acting on the vectors of the complex Leech lattice vectors perpendicular to the distinguished hexagonal section. The $\mathrm{M}_{11}$ group was discovered in the $19^{\text {th }}$ century by the mathematical physicist Emil Mathieu, and was the first in the series of discoveries of sporadic finite simple groups that culminated in 1981 with Griess' construction of the Monster sporadic group [12] [13]. An intriguing aspect of our model for space-times with matter is that our theory seems to be intimately connected with the mathematical structure of several of the sporadic finite groups.

In Section 2, we review the gauged nonlinear Schrodinger equation theory of Einstein spaces. In Section 3, we exhibit a matrix gauged nonlinear Schrodinger equation that we believe provides a framework for understanding the quantum nature of space-times with matter. We also exhibit the matrix generalization of the classical "Heavenly" equation for the Kahler potential for a self-dual or anti-self dual Einstein space. The solutions of the matrix self-duality condition provide the building blocks for a theory of space-time that amounts to a superfluid theory of superfluids. In Section 4, we comment on the possibility that our emergent model for space-time not only provides a natural explanation for the appearance of a cosmological constant at the time of the big bang, but also allows for the appearance of a vacuum energy during the gravitational collapse of massive objects.

\section{Non-Linear Schrodinger Equation for Einstein Spaces}

The coherent state wave function for a 2-dimensional quantum fluid of anyons interacting via both a point-like interaction and Chern-Simons gauge potentials satisfies the non-linear Schrodinger equation [15]: 


$$
i \hbar \frac{\partial \psi}{\partial t}=-\frac{1}{2 m} D^{2} \psi+e A_{0} \psi-g|\psi|^{2} \psi
$$

where $D_{\alpha}=\partial_{\alpha}-i(e / \hbar c) A_{\alpha}$ and $m$ is a mass parameter. The gauge fields $A_{0}$ and $A_{\alpha}$ do not satisfy Maxwell's equations, but instead are determined self-consistently from the equations for Chern-Simons electrodynamics in $2+1$ dimensions. In the presence of a uniform 2-dimensional electric field $E$ the current has the same form as the Hall current for a magnetic field perpendicular to the plane

$$
j_{\alpha \beta}=\sigma_{H} \varepsilon_{\alpha \beta \gamma} E_{\gamma}
$$

where $\sigma_{H}$ is the "Hall conductivity". Neglecting spatial variations in the electric field, the usual Guass' law will be replaced by the Chern-Simons equation

$$
B=-\frac{\rho}{\kappa}
$$

where $B$ is the strength of an effective magnetic field whose direction is perpendicular to the layer, $\rho$ is the charge per unit area, and $1 /|\kappa|$ is an inverse length with $\sigma_{H}=\kappa$. In model where charged particles hop on a hexagonal lattice the nonlinear term with coefficient $g$ represents the effect of spin orbit coupling. It was shown in ref. [16] that the time independent version of Equation (1) in conjunction with Equations (1) \& (2) can be solved analytically if one assumes that

$$
g= \pm e^{2} \hbar / m c \kappa
$$

The ground state solution of Equation (1) contains solitons with vortex-like currents and two units of quantized magnetic flux attached to every carrier. The two signs for $g$ correspond to solutions where the vorticity of all the solitons is either up or down.

In 1991, the author introduced the idea [2] of extending Equations (1)-(3) spatial dimensions by replacing the 2-dimensional complex plane with a stack of $\mathrm{N}$ such planes and the scalar wave function with an $\mathrm{SU}(\mathrm{N})$ matrix:

$$
i \hbar \frac{\partial \Phi}{\partial t}=-\frac{1}{2 m} D^{2} \Phi+e\left[A_{0}, \Phi\right]-g\left[\left[\Phi^{*}, \Phi\right], \Phi\right]
$$

where the wave function $\Phi$ and potentials $A_{0}$ and $A_{i}$ are now SU(N) matrices, and $D \equiv \nabla-i(e / \hbar c)[A$, . Promoting the scalar wave function $\psi(z)$ to an $\mathrm{SU}(\mathrm{N})$ matrix wave function $\Phi(z)$ is a way to take into account into account inter-layer interactions and tunneling. The magnetic field

$B_{\text {eff }}=\partial_{x} A_{y}-\partial_{y} A_{x}+\left[A_{x}, A_{y}\right]$ seen by charged carriers in 2-dimensions is now a diagonal matrix

$$
B_{e f f}=-\frac{e}{\kappa}\left[\Phi^{*}, \Phi\right]
$$

The in-plane electric field $E_{\alpha}$ will also be a diagonal matrix satisfying the Hall effect equation:

$$
E_{\alpha}=-\frac{1}{\kappa} \varepsilon_{\alpha \beta} j_{\beta}
$$


where $\alpha, \beta=1,2, \varepsilon_{\alpha \beta}=-\varepsilon_{\beta \alpha}$, and

$$
j_{\alpha}=(\hbar / 2 m i)\left(\left[\Phi^{*}, D_{\alpha} \Phi\right]-\left[D_{\alpha} \Phi^{*}, \Phi\right]\right)
$$

is the in-plane current. It had previously been shown [16] that time independent analytic solutions to Equations (5)-(7) can be found for any value of $N$ if Equation (4) is satisfied. These analytic solutions represent zero energy ground states and satisfy the 2-dimensional self-duality condition $D_{\alpha} \Phi= \pm i \varepsilon_{\alpha \beta} D_{\beta} \Phi$.

In the limit $N \rightarrow \infty$ the analytic solutions of Equation (5) take a particularly simple form such that the effective magnetic field seen by the th soliton has the simple form:

$$
B_{j}= \pm \frac{\hbar c}{e} \sum_{k} \nabla_{k}\left|X_{j}-X_{k}\right|
$$

where $X \equiv(z, u)$ is now a 3-dimensional coordinate encoding both the position $z=x+i y$ of a soliton within a layer and the height $u$ of the layer. In this solution the vortex-like solitons present in the solution for a single layer have become monopole-like objects, which were christened "chirons" in Ref. [2]. The ground state corresponding to (8) has zero energy and the wave function has the form [3] [4]

$$
\Psi=f(w) \prod_{k>j}^{\infty}\left[\frac{R_{j k}+U_{j k}}{R_{j k}-U_{j k}}\right]^{1 / 2}
$$

where $R_{j k}^{2}=U_{j k}^{2}+4\left(z_{j}-z_{k}\right)\left(\bar{z}_{j}-\bar{z}_{k}\right), \quad U_{j k}=u_{j}-u_{k}$, and $f$ is an entire function of the $\left\{\bar{z}_{i}\right\}$ in the self-dual case and $\left\{z_{i}\right\}$ in the anti-self-dual case. Writing the product on the r.h.s. of Equation $(10)$ as $\exp (S)$ defines an effective action for a gas of chirons:

$$
S=\frac{1}{2} \sum_{j} \ln \frac{R_{j}+u-u_{j}}{R_{j}-u+u_{j}}
$$

where $R_{j}^{2}=\left(u-u_{j}\right)^{2}+4\left(z-z_{j}\right)\left(\bar{z}-\bar{z}_{j}\right)$. The wave function (10) resembles in some respects Laughlin's wave function for the fractional quantum Hall effect; for example moving the $z$ coordinate of a chiron around the position of another chiron in a different layer changes $S$ by $i \pi$ [2] [3]. However, in contrast with the fractional quantum Hall effect, there are two distinct degenerate ground states corresponding to the self-dual and anti-self-dual solutions for Equation (5), reminiscent of the Kramers pairs in systems with time reversal symmetry. It was the motivation for the suggestion [3] that these two solutions can be combined to yield a model for empty space-time.

Actually the effective action (11) for chirons already suggests a connection with the Kosterlitz-Thouless condensation of vortex and anti-vortex pairs in the 2-dimensional XY model [17]. It is an elementary identity that the right hand side of (10) can be rewritten in the form

$$
S=\sum_{i} \pm \tanh ^{-1}\left(\frac{u-u_{i}}{R_{i}}\right)
$$


which is similar in form to a configuration of 2-dimensional XY vortices. In the $\mathrm{XY}$ model the phase variations in a 2-dimensional condensate can be described by a partition function of the form

$$
Z=\int_{0}^{2 \pi} D \Theta \exp \left[-\frac{K}{2} \int \mathrm{d}^{2} \xi \frac{\partial \Theta}{\partial \xi_{i}} \frac{\partial \Theta}{\partial \xi_{i}}\right]
$$

where $\Theta$ is a periodic coordinate whose period is $2 \pi$ and $K$ is a constant. It can be shown that a discrete version of this theory interpolates between the low and high temperature phases of the XY model. Indeed evaluating the exponential in (13) for a configuration of vortices yields the partition function for a 2-D Coulomb gas. On the other hand substituting the chiron effective action (12) into the exponential in (13) yields:

$$
\exp -\pi K\left\lfloor\sum_{i \neq j} m_{i} m_{j} \ln \frac{R_{i j}}{\left|z_{i}-z_{j}\right|}\right\rfloor
$$

Expression (14) illustrates why Einstein spaces such as flat Minkowski space-time can also be viewed as a condensation of self-dual and anti-self-dual chirons. Although the pairing of self-dual and anti-self-dual solitons of the Einstein equations must classically be defined using two separate 2-dimensional spaces (the "ambi-twister" construction of Einstein spaces), it is also possible [17] to regard (14) as defining the phase of a quantum state consisting of a superposition of coherent quantum states corresponding to the self-dual and anti-self dual solutions of Equation (5). This construction amounts to a matrix generalization of the Pitaevskii, theory of superfluids [18], and suggests that the idea introduced in ref. [10] of using a non-linear Schrodinger equation to represent the quantum dynamics of space-times deformed by matter may be on the right track.

\section{Extension to Include Matter}

In this section we will outline how the "superfluid" description of Einstein spaces introduced in Section 2 might be extended to space-times with matter and/or vacuum fields. Since the solutions of the 2-dimensional nonlinear Schrodinger equation that were used to construct Einstein spaces were either holomorphic or anti-holomorphic functions, we will assume that it is actually the complex Leech lattice, which is a 12-dimensional lattice whose coordinates are Eisenstein integers, rather than the real integers that is the natural setting for our theory.

Our construction begins by replacing the complex plane used in the construction of Einstein spaces with a distinguished hexagonal lattice section of the Leech lattice. The particular hexagonal section we will use corresponds to the Eisenstein vector $\left(3 \sqrt{3} \mathrm{i}, 0^{11}\right)$. The full 12-dimensional symmetry of complex Leech lattice will be broken by our use of this distinguished hexagonal lattice. The automorphism group of the Leech lattice, $0 \bullet$, is transitive on all the vertices of the Leech polytrope, but has a subgroup, $2^{12} \mathrm{M}_{24}$, that preserves the shape of the complex Leech polytrope, where $2^{12}$ is a group of special involutions of the 
Leech lattice, and $\mathrm{M}_{24}$ is another of the exceptional permutation groups discovered by Mathieu [12]. If a 2-dimensional section of the real Leech lattice is considered fixed, then the automorphism group $0 \bullet$ of the Leech lattice is reduced to a subgroup $2^{10} \mathrm{M}_{22}$, where $2^{10}$ is again a group of special involutions, while $\mathrm{M}_{22}$ is the subgroup of $\mathrm{M}_{24}$ that fixes two letters. The subgroup of $0 \bullet$ which preserves the structure of the complex Leech lattice is 6. Suz, where Suz is the Suzuki sporadic permutation group [13], while the subgroup which fixes our choice for the distinguished fixed direction is $2 \times 3^{6}: M_{11}$, where $M_{11}$ is the Mathieu permutation group acting on the vectors of the complex Leech polytrope that are perpendicular to the fixed direction.

The miracle of life in the context of our construction of space-times with matter is that the symmetry group $M_{11}$ acting on the vectors of the complex Leech polytrope has orbits corresponding to vectors with shapes familiar from the theory of Lie groups. This allows us to represent the $M_{11}$ symmetry of the matter degrees of freedom in our theory by introducing a gauge field into the nonlinear Schrodinger equation. For example, there is an orbit consisting of the vectors with 2 nonzero components different from the fixed direction. $\mathrm{M}_{11}$ acts on these vectors in the same way that the $11 \times 10 / 2=55$ generators of $\mathrm{SO}(11)$ would act on a 2 -form gauge field $B_{\mu \nu}$ in 11 dimensions. What is especially noteworthy for our purposes is that the action of $\mathrm{M}_{11}$ within this orbit be lifted to a continuous $\mathrm{SO}(11)$ gauge transformation by exponentiation.

Another interesting orbit of $M_{11}$ emerges from the amusing fact that the Mathieu group $\mathrm{M}_{12}$ can be represented as the 5-cycle twists of the vertices of a 3-dimensional icosahedron around each vertex together with an inversion of the icosahedron vertices [12]. This provides a nice picture for how $M_{11}$ transforms complex Leech polytrope vectors with different shapes. For example, it is immediately evident that the action of $\mathrm{M}_{11}$ on the Leech polytrope vectors representing a 2-form gauge field $B_{\mu \nu}$ corresponds to rotations of the icosahedron that fix one axis of the icosahedron. It is also straightforward to show that the subgroup of $\mathrm{M}_{11}$ corresponding to transformations of the 36 pairs of icosahedron vertices whose 5-cycles leave one vertex fixed can identified with 2 copies of the generators of an $\mathrm{E}_{6}$ gauge symmetry. The remarkable coincidence that the $\mathrm{M}_{11}$ symmetry acting on the complex Leech polytrope with one direction fixed can identified with the generators of well known Lie groups allow us to introduce nonabelian gauge fields to represent the Leech lattice symmetries of our theory. These nonabelian gauge fields are a natural generalization of the U(1) Chern-Simons gauge field that we used to construct Einstein space-times, and points us in the direction of pursuing the same sort of strategy for constructing emergent space-times with matter that we used to construct Einstein spaces.

The bare bones for our model is the matrix nonlinear Schrodinger equation in 2-dimensions,

$$
H=\frac{1}{2 m} \int \mathrm{d}^{2} x\left(\left(D_{i} \Psi\right)^{*}\left(D_{i} \Psi\right)+g\left(\left[\Psi^{\uparrow}, \Psi\right]\right)^{2}\right)
$$


where $i=1,2$ and

$$
D_{\alpha}=\partial_{\alpha}+e\left[A_{\alpha},\right.
$$

where $A_{\alpha}$ is an $11 \times 11$ matrix describing the actions of the 2 -form and Yang-Mills gauge fields obtained by restricting the coordinates of the Leech lattice to lie in the 11 dimensions orthogonal to the distinguished 2-dimensional section. As was the case for the nonlinear Schrodinger theory of Einstein spaces, the gauge field $A_{\alpha}$ that enters Equation (16) is not an independent variable, but will be entirely determined by the matrix wave function. An elegant way to represent this interdependence is to introduce a generalized matrix wave function $W_{1,2} \equiv A_{1,2}, \quad \Psi \equiv W_{3}+i W_{4}, \quad \Psi^{*} \equiv W_{3}-i W_{4}$. The Chern-Simons and self-duality constraints can then be combined into a single constraint for $W_{\dot{\alpha}}$ :

$$
G_{\alpha \beta} \equiv \partial_{\alpha} W_{\beta}-\partial_{\beta} W_{\alpha}+\left[W_{\alpha}, W_{\beta}\right]=\frac{1}{2} \varepsilon_{\alpha \beta \delta \gamma} G^{\delta \gamma}
$$

where $\alpha, \beta=1,2,3,4$. The Chern-Simons constraints can also be introduced by adding a topological term to the Lagrangian for the system (15):

$$
L_{C S}=\frac{\kappa}{2} \varepsilon^{\alpha \beta \gamma} \operatorname{Tr}\left(A_{\alpha} \partial_{\beta} A_{\gamma}+\frac{2}{3} A_{\alpha} A_{\beta} A_{\gamma}\right)
$$

The fact that the Chern-Simons and self-duality conditions for either the $\mathrm{SU}(\mathrm{N})$ case discussed in ref. [16] or the nonabelian gauge fields associated with the orbits of $\mathrm{M}_{11}$ are similar in form means that we can pursue similar strategies for solving these constraints. In particular if we can represent the wavefunction as a linear combination of raising and lowering operators for the $\mathrm{S}(11)$ or $\mathrm{E}_{6}$ Lie algebras:

$$
\partial_{z} \partial_{\bar{z}} \phi_{a}=-\frac{e}{\kappa} \sum_{b} K_{a b} \mathrm{e}^{\phi_{b}}
$$

where $K_{a b}$ is the Cartan matrix for the $\mathrm{SO}(11)$ and $\mathrm{E}_{6}$ nonabelian Lie algebras associated with the orbits of $M_{11}$. This equation generalizes the "Heavenly Equation" for the Kahler potential for a self-dual Einstein space [1]. The appearance of a 2-form gauge field degree of freedom associated with Yang-Mills degrees of freedom is intriguing from the point of view that a Lagrangian involving a 2 -form gauge field and Yang-Mills gauge field strengths plays an important role in cancelling the hexagon anomaly of Yang-Mills theories in superstring theories [19].

Although there may be other possible choices for the interaction Lagrangian, our choice is motivated by the observation that the Yang-Mills degrees of freedom are represented by the vertices of the complex Leech polytope with one axis fixed with shape $\left(\alpha^{5}, 0^{6}\right)$, where $\alpha$ is a cube-root of -1 . The number of such vertices is determined by the $(4,5,11)$ Witt design, which underlies the $M_{11}$ permutation symmetry [20]. Apart from the 3-fold involutions associated with multiplying each coordinate by a cube root of -1 , these vertices can be generated by associating each distinguished 4 -set in the Witt design one of three $3 \times 3$ matrices representing the $3 \times 36$ generators for 3 copies of the complex $\mathrm{E}_{6}$ Lie algebra. 
In a similar way, 2-form gauge fields $B_{a i}$ can be generated by two 11 dimensional vectors. If we ignore the lattice structure of the hexagonal section then we can expect that holomorphic and anti-holomorphic matrix functions will play a central role in our new theory of space-time. In the context of the complex Leech lattice the wave function and Chern-Simons gauge potential will be $11 \times 11 \mathrm{ma}$ trices, which will represent extra degrees of freedom compared to metric tensor of classical general relativity.

Our model follows the Einstein space construction by insisting that the dynamics of space-time is determined by a Chern-Simons gauge field in $2+1$ real dimensions, except the Chern-Simons gauge field is now a complex matrix belonging to the adjoint representation of a Lie algebra. We are thus led to the use a topological interaction Lagrangian that recognizes the difference between the distinguished hexagonal section of the Leech lattice used to represent the 2 of the dimensions of ordinary space-time and the 11 dimensions representing internal degrees of freedom. Actually the author anticipated some time ago [21] that a fundamental theory of gravity and elementary particles might involve a topological form in 13 dimensions. We also note that it was Richard Slansky [22] who originally suggested that the Yang-Mills gauge symmetry in a unified theory of elementary particles might be $\mathrm{E}_{6}$.

When time is added to the nonlinear matrix equation the wave function will depend not only on position within the distinguished 2-dimensional section of the Leech lattice, but also time. However, as in our studies of Einstein spaces, we will initially be mainly interested in solutions which are time independent; i.e. solutions corresponding to a ground state with zero energy. Remarkably the numbers of internal bosonic and fermionic degrees of freedom of our theory exactly match, even though the corresponding fields are defined in 24 dimensions. This means that at the level of $2+1$ dimensions the contribution of zero point fluctuations of bosonic or fermionic superfluid modes to the ground state energy will vanish. However, when time and a $3^{\text {rd }}$ spatial dimension are added to construct a theory of space-time in $3+1$ dimensions, the contributions of the bosonic and fermionic degrees of freedom to the ground state energy will in general not exactly cancel each other. Consequently in our emergent model for space-time the vacuum energy will in general not be zero.

\section{Conclusions}

In conclusion, we believe that the equations proposed in Section 3 fill a void that arises from the fact that neither classical general relativity nor superstring theory appear to be capable of explaining a number of pressing astrophysical puzzles. These include the physical nature of the compact objects formed by gravitational collapse, the physical nature of the big bang, and under what circumstances a vacuum energy may appear. We note that classical general relativity does not provide any guidance as to when a vacuum energy may appear because classical general relativity does not take into account the quantum nature of the vacuum state. 
Some time ago the author and Nick Manton pointed out [23] that a "geometric" Higgs potential could appear in the 3-dimensions of ordinary space-time as a result of a topologically non-trivial configuration of vacuum gauge fields in extra dimensions. In the context of superstring theory a similar mechanism allows for the appearance of a negative vacuum energy [24], while a somewhat different mechanism [25] allows leads to metastable de Sitter vacuums. However, it is not yet clear how the proposed superstring mechanisms for generating a vacuum energy relate to the nature of compact objects or the origin of the big bang. Our original superfluid model for compact objects [10] relied on Lemaitre's seminal observation [26] that the appearance of a vacuum energy can prevent a (spherically symmetric) object from collapsing to a singularity. Indeed, the appearance of a positive vacuum energy during gravitational collapse [27] is the only mechanism known for preventing continuous collapse to a singularity. To date though there has been no claim from the string theory community that gravitational collapse of matter can lead to the appearance of a positive vacuum energy.

In general the appearance of a positive vacuum energy is only possible if supersymmetry is broken. This doesn't appear to be a necessity in superstring theory, but as noted above the graviton and matter degrees of freedom in our superfluid theory of space-time appear in different representations of the Leech lattice automorphism group, and therefore it is quite natural for supersymmetry to be broken in situations where the gravitational and matter degrees of freedom are strongly coupled. Indeed our theory offers a direct path to not only explaining why black hole singularities are avoided during gravitational collapse, but what role the appearance of a positive vacuum energy may play in the big bang. Indeed one would expect that in the context of the big bang our theory would predict that positive vacuum energies corresponding to the scale supersymmetry breaking (i.e. $\sim 1 \mathrm{TeV}^{4}$ ) would make an appearance.

Beyond these observations that our superfluid theory of space-time appears to be better prepared to address fundamental astrophysical puzzles we will leave for the future the challenge of finding exact solutions of our matrix non-linear Schrodinger equation.

\section{Acknowledgements}

The author is very grateful for discussions with Bernard Grossman, Pawel Mazur, Emil Mottola, Samuel Braunstein, and Jim Barbieri.

\section{References}

[1] Plebankski, J. (1975) Journal of Mathematical Physics, 16, 2395. https://doi.org/10.1063/1.522505

[2] Chapline, G. and Yamagishi, K. (1991) Physical Review Letters, 66, 3046. https://doi.org/10.1103/PhysRevLett.66.3064

[3] Chapline, G. (1992) Modern Physics Letters A, 7, 1959. https://doi.org/10.1142/S0217732392001671 
[4] Chapline, G. (1993) Anyons and Coherent Sates for Gravitons. In: Yang, C.N., Gee, M.L. and Zhou, X.W., Eds., Proceedings of the XXI International Conference on Differential Geometric Methods in Theoretical Physics, World Scientific, Singapore, 455.

[5] Chapline, G. (1992) Information Flow in Quantum Mechanics: The Quantum Maxwell Demon. In: Black, T., et al., Eds., Proceedings of Santa Fe Conference on the Foundations of Quantum Mechanics, World Scientific, Singapore, 255.

[6] Mazur, P.O. (1997) AIP Conference Proceedings, 415, 299. https://doi.org/10.1063/1.54455

[7] Boulware, D. (1975) Physical Review D, 11, 1404. https://doi.org/10.1103/PhysRevD.11.1404

[8] Braunstein, S.L., Pirandola, S. and Zyczkowski, K. (2013) Physical Review Letters, 110, 101301. https://doi.org/10.1103/PhysRevLett.110.101301

[9] Almheiri, A., Marolf, D., Polchinski, J. and Sully, S. (2013) Journal of High Energy Physics, 2, 62. https://doi.org/10.1007/JHEP02(2013)062

[10] Chapline, G., Hohlfeld, E., Laughlin, R. and Santiago, D. (2001) Philosophical Magazine $B, \mathbf{8 1}, 235$. https://doi.org/10.1080/13642810108221981

[11] Leech, J. (1967) Canadian Journal of Mathematics, 19, 251. https://doi.org/10.4153/CJM-1967-017-0

[12] Conway, J.H. and Sloane, N.J.A. (1988) Sphere Packings, Lattices, and Groups. Springer-Verlag. https://doi.org/10.1007/978-1-4757-2016-7

[13] Chapline, G. and Manton, N. (1983) Physics Letters B, 120, 105-109. https://doi.org/10.1016/0370-2693(83)90633-0

[14] Conway, J., et al. (1985) Atlas of Finite Simple Groups. Clarendon Press, Oxford.

[15] Voit, J. (1990) Physical Review Letters, 64, 323. https://doi.org/10.1103/PhysRevLett.64.323

[16] Grossman, B. (1990) Physical Review Letters, 65, 3230. https://doi.org/10.1103/PhysRevLett.65.3230

[17] Chapline, G. (2006) Philosophical Magazine, 86, 1201 https://doi.org/10.1080/14786430500197405

[18] Pitaevskii, L.P. (1961) Soviet Physics JETP, 13, 451.

[19] Green, M.B. and Schwarz, J.H. (1984) Physics Letters B, 149, 117. https://doi.org/10.1016/0370-2693(84)91565-X

[20] Witt, E. (1938) Abhandlungen aus dem Mathematischen Seminar der Universität Hamburg, 12, 256-264. https://doi.org/10.1007/BF02948947

[21] Chapline, G. (1985) Physics Letters B, 158, 393-396. https://doi.org/10.1016/0370-2693(85)90439-3

[22] Slansky, R. (1981) Physics Reports, 79, 1.

[23] Chapline, G. and Manton, N. (1981) Nuclear Physics B, 184, 391-405. https://doi.org/10.1016/0550-3213(81)90226-1

[24] Giddings, S., Kachru, S. and Polchinski, J. (2002) Physical Review D, 9, 106006.

[25] Ibanez, L. and Uranga, A. (2012) String Theory and Paticle Physics. Cambridge U. Press.

[26] Lemaître, A.G. (1997) General Relativity and Gravtation, 29, 641-680. https://doi.org/10.1023/A:1018855621348

[27] Chapline, G. and Barbieri, J. (2014) International Journal of Modern Physics D, 23, 1450025. https://doi.org/10.1142/S0218271814500254 